\title{
PO3-9-9
}

Poster session

\section{Transcriptome profiling of an extensively drug-resistant and carbapenemase- producing hypervirulent Klebsiella pneumoniae strain identifies novel regulatory mechanisms under antibiotics treatments}

\author{
Yen-Hua Huang ${ }^{1}$, Yi-Tsung Lin², Syun-Wun Liang ${ }^{3}$, Chung-En Ni \\ ${ }^{1}$ Institute of Biomedical Informatics, National Yang-Ming University, Taiwan, ${ }^{2}$ Division of Infectious Diseases, \\ Department of Medicine, Taipei Veterans General Hospital and Institute of Emergency and Critical Care Medicine, \\ National Yang-Ming University, Taiwan, ${ }^{3}$ Center for Systems and Synthetic Biology, National Yang-Ming University, \\ Taiwan
}

Background: During the past few years, Klebsiella pneumonia has become a threat to human health because emerging strains could be both multidrug resistant and hypervirulent. In this project, we explored the regulatory mechanisms underlying the drug resistance of a carbapenem-resistant hypervirulent (CR-hv) Klebsiella pneumoniae strain. The specific aims are as follows: 1) generating a complete reference genome; 2) finding differentially expressed genes and operons; 3) finding differentially expressed antisense transcripts and sRNAs implicated in stress response.

Materials and Methods: The whole genome sequencing of a CR-hv KP isolate, TVGHCRE225, was performed by using RSII system, Pacific Bioscience of California, Inc. To investigate the transcriptome changes of TVGHCRE225 under the treatments of antibiotics, this KP strain was cultured in separated media containing colistin and tigecycline, respectively. Then eight samples collected at the mid-log and log phases were taken to perform RNA-seq and sRNA-seq experiments for transcriptome profiling. Additionally, using bioinformatics approaches we predicted and annotated sRNAs in all the non-protein coding regions, including the antisense strand of known protein-coding genes.

Results: The assembled genome for TVGHCRE225 consists of the 5.5-Mbp chromosome and three plasmids. 5,895 genes were predicted and 65 of them were hypothetical. One plasmid is pLVPK-like, which harbors capsular-gene transcription factors, rmpA and rmpA2, and a ferric aerobactin receptor, iutA, providing an explanation to the high virulence of this KP isolate. Additionally, $\sim 300$ novel non-coding RNA genes and 50 antisense transcripts were identified from the RNAseq and sRNA-seq results. Noticeably, under the treatments of two different antibiotics, respectively, distinct sets of differentially expressed genes (DEGs) were identified, with only a few genes co-occurred. Changes over the operon structures with varied member genes were observed and such structural changes could be categorized into merged/extended, shortened, and mixed types.

Conclusions: Those DEGs along with the structurally changed operons under antibiotics treatment were implicated in a diverse range of biological pathways, not just the well-known stress responses. Our findings suggest that antibiotics resistance of bacteria might be highly regulated where a number of noncoding RNAs such as antisense transcripts and small RNAs may play critical roles. 